\title{
Observation of a chiral wave function in the twofold-degenerate quadruple Weyl system BaPtGe
}

\author{
Haoxiang Li, ${ }^{1, *}$ Tiantian Zhang, ${ }^{2,3,{ }^{*}, \dagger}$ A. Said,${ }^{4, *}$ Y. Fu, ${ }^{5}$ G. Fabbris $\odot,{ }^{4}$ D. G. Mazzone $\odot,{ }^{6}$ J. Zhang, ${ }^{1}$ J. Lapano, ${ }^{1}$ \\ H. N. Lee $\odot,{ }^{1}$ H. C. Lei,${ }^{5,+}$ M. P. M. Dean $\odot,{ }^{7}$ S. Murakami $\odot,{ }^{2,3}$ and H. Miao $\odot^{1, \S}$ \\ ${ }^{1}$ Materials Science and Technology Division, Oak Ridge National Laboratory, Oak Ridge, Tennessee 37831, USA \\ ${ }^{2}$ Department of Physics, Tokyo Institute of Technology, Okayama, Meguro-ku, Tokyo 152-8551, Japan \\ ${ }^{3}$ Tokodai Institute for Element Strategy, Tokyo Institute of Technology, Nagatsuta, Midori-ku, Yokohama, Kanagawa 226-8503, Japan \\ ${ }^{4}$ Advanced Photon Source, Argonne National Laboratory, Argonne, Illinois 60439, USA \\ ${ }^{5}$ Department of Physics and Beijing Key Laboratory of Opto-Electronic Functional Materials and Micro-devices, \\ Renmin University of China, Beijing, China \\ ${ }^{6}$ Laboratory for Neutron Scattering and Imaging, Paul Scherrer Institut, CH-5232 Villigen, Switzerland \\ ${ }^{7}$ Condensed Matter Physics and Materials Science Department, Brookhaven National Laboratory, Upton, New York 11973, USA
}

(Received 29 October 2020; accepted 16 April 2021; published 4 May 2021)

\begin{abstract}
Topological states in quantum materials are defined by bulk wave functions that possess nontrivial topological invariants. While edge modes are widely presented as signatures of nontrivial topology, how bulk wave functions can manifest explicitly topological properties remains unresolved. Here, using high-resolution inelastic x-ray spectroscopy (IXS) combined with first principles calculations, we report experimental signatures of chiral wave functions in the bulk phonon spectrum of BaPtGe, which we show to host a previously undiscovered twofolddegenerate quadruple Weyl node. The chirality of the degenerate phononic wave function yields a nontrivial phonon dynamical structure factor, $S(\mathbf{Q}, \omega)$, along high-symmetry directions, that is in excellent agreement with numerical and model calculations. Our results establish IXS as a powerful tool to uncover topological wave functions, providing a key missing ingredient in the study of topological quantum matter.
\end{abstract}

DOI: 10.1103/PhysRevB.103.184301

In high-energy physics, chirality is an inherent quantum property of fundamental particles that originates from the Poincaré group. In condensed matter systems, however, chiral quasiparticles (QPs) can only emerge from low-energy effective models that must respect crystalline symmetries. Well-known examples are Weyl fermions in topological semimetals [1-6] and Weyl bosons in chiral lattices and optical and spin systems [7-15]. Although these chiral QPs emerge from the materials' bulk wave functions, existing experimental studies have focused either on the consequences of these quasiparticles, such as Fermi-arc surface states [16-25] and chiral anomalies [26-30], or argued based on a comparison of eigenvalues (band dispersions) between experiment and theory $[12,13,31]$, leaving the bulk wave function unresolved. This is unfortunate as the information of topological invariants, such as the Chern number, $C$, and the chirality of QPs, is carried by the bulk wave functions. Filling this gap is challenging as it requires a bulk sensitive spectroscopic probe that has high energy and momentum resolutions and a well-defined scattering cross section that can be analytically or numerically calculated. In this article, we demonstrate that high-resolution inelastic x-ray scattering (IXS) combined with

\footnotetext{
${ }^{*}$ These authors contributed equally to this work.

${ }^{\dagger}$ ttzhang@stat.phys.titech.ac.jp

\#hlei@ruc.edu.cn

§miaoh@ornl.gov
}

first-principles calculations provide direct evidence of topological phononic wave functions in the chiral cubic crystal BaPtGe.

A fascinating property of $\mathrm{BaPtGe}$ is that it is predicted to host multiple chiral topological states, including a new and undiscovered twofold-degenerate quadruple Weyl node (TQW) [32] that can only be realized in bosonic excitations, like phonons, or electronic structures without spin-orbit coupling. Unlike mechanical metamaterials [19,23,25], phonons in crystalline materials are true quantum objects and correlate with electronic and magnetic excitations [7,33]. Past work has tended to assume that a Weyl node with large $C$ requires a large band degeneracy, $n$ [6]. Indeed only $C \leqslant n$ has been experimentally observed to date. A remarkable feature of TQW is that a large Chern number, $C=4$, is realized in the smallest possible band degeneracy, $n=2$. This unusual topological structure is induced by the chiral, cubic, and timereversal symmetry of BaPtGe (space group $P 2{ }_{1} 3$ \#198) shown in Figs. 1(a) and 1(d) that enforce certain Weyl nodes at the time-reversal-invariant $\Gamma$ point. The low-energy effective Hamiltonian near the $\Gamma$ point for the two modes that make up a TQW point is

$$
H(\mathbf{q})=\left(\begin{array}{cc}
A q_{x} q_{y} q_{z} & B^{*}\left(q_{x}^{2}+\omega^{2} q_{y}^{2}+\omega q_{z}^{2}\right) \\
B\left(q_{x}^{2}+\omega^{2} q_{y}^{2}+\omega q_{z}^{2}\right) & -A q_{x} q_{y} q_{z}
\end{array}\right),
$$

where $\omega=e^{2 \pi i / 3}, A$ is a real constant, and $B$ is a complex constant. $\mathbf{q}$ is the reduced momentum transfer in the first Brillouin zone. The leading $q^{2}$ term distinguishes TQW from all 
(a)
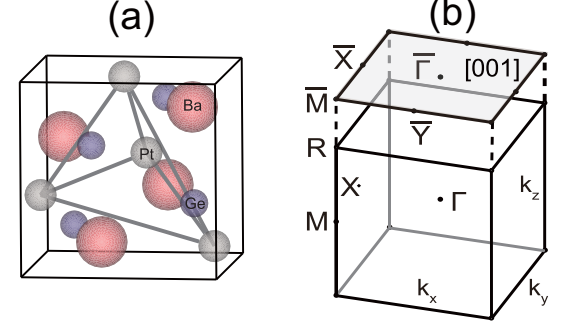

(d)
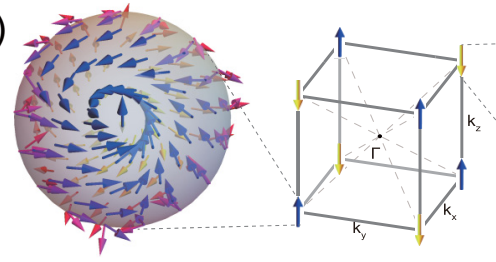

(c)
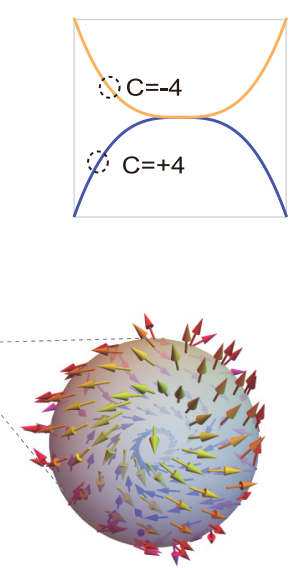

(e)

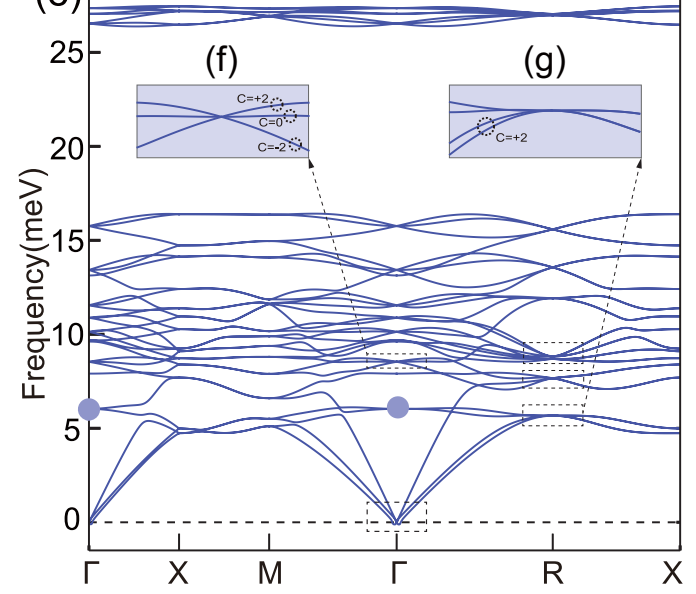

FIG. 1. Twofold-degenerate quadruple Weyl node and chiral wave functions. (a), (b) Crystal structure and Brillouin zone (BZ) of BaPtGe, respectively. The high-symmetry points in the three-dimensional BZ and the projected two-dimensional BZ are shown in (b). (c) TQW node with $C=+4,-4$ for each band. (d) Pseudospin derived from Eq. (1) at the eight symmetry-related $R$ points. Colored arrows represent pseudospin directions. Zoomed pseudospin textures near time-reveal-related momenta $(0.5,-0.5,-0.5)$ and $(-0.5,0.5,0.5)$ in reciprocal lattice units (r.l.u.) are showing opposite chirality. (e) DFT-calculated phonon dispersion of BaPtGe. Solid circles at the $\Gamma$ points correspond to the theoretically predicted TQW. Dashed rectangles at the $\Gamma$ and $R$ points are corresponding to threefold and fourfold double Weyl nodes, which are zoomed in (f)- (g).

other Weyl nodes proposed in previous studies [see Fig. 1(c)] [6]. For instance, the two modes that arise from diagonalizing Eq. (1) have special pseudospin properties (see more detail discussions in the Supplemental Material [34]). Contours encircling the TQW point involve four windings of the pseudospin, reflecting the large $C=4$. In Fig. 1(d), we show pseudospin orientations at the eight $R( \pm 0.5, \pm 0.5, \pm 0.5)$ points. At these high symmetry locations, pseudospin points along the $\pm z$-direction with its sign [marked as blue or yellow in Fig. 1(d)] determined by the sign of $q_{x} q_{y} q_{z}$. Consequently, the phonon wave functions of BaPtGe that make up the TQW point involve opposite out-of-phase chiral atomic motions around time-reversal related $R$ points either side of the $\Gamma$ point. In addition to the TQW, BaPtGe also features other topological structures, including the threefold double Weyl phonon with $n=3$ and $C=2$ at the $\Gamma$ point and fourfold double Weyl phonon with $n=4$ and $C=2$ at the $R$ point $[7,12]$. All of them have chiral bulk wave functions [7,12,15,32]. In Fig. 1(e), we show the density functional theory (DFT) calculation of the BaPtGe phonon dispersion along high symmetry directions. A low-energy TQW is identified near $6 \mathrm{meV}$ at the $\Gamma$ point (purple circle), which involves primarily atomic vibrations of the heaviest element Pt. The threefold and fourfold double Weyl nodes, that have been explored in both fermionic and bosonic systems [12,35], are marked by dashed squares at the $\Gamma$ and $R$ points, respectively.

We now illustrate how IXS probes the phononic wave functions. Under the harmonic approximation, the phonon excitations are described by

$$
\sum_{d^{\prime}} D_{d d^{\prime}}(\mathbf{q}) \mathbf{e}_{d}^{j}(\mathbf{q})=\omega_{j q}^{2} \mathbf{e}_{d}^{j}(\mathbf{q})
$$

where $D(\mathbf{q})$ is the phonon dynamical matrix, and $\mathbf{q}$ is the reduced momentum transfer in the first Brillouin zone. $\omega_{j q}$ and $\mathbf{e}_{d}^{j}(\mathbf{q})=\left[e_{d x}^{j}(\mathbf{q}), e_{d y}^{j}(\mathbf{q}), e_{d z}^{j}(\mathbf{q})\right]$ are the eigenvalue and wave function of the phonon mode $j$ at atom $d$, respectively. IXS measures the bulk phonon dynamical structure factor

$$
\begin{aligned}
S(\mathbf{Q}= & \mathbf{q}+\mathbf{G}, \omega) \\
= & C_{0} \sum_{j} \frac{1}{\omega_{q j}}\left|\sum_{d} \frac{f_{d}}{\sqrt{M_{d}}} \mathbf{Q} \cdot \mathbf{e}_{d}^{j}(\mathbf{q}) \exp \left(i \mathbf{Q} \cdot \mathbf{r}_{d}\right)\right|^{2} \\
& \times\left\{\begin{array}{c}
\left\langle n_{\omega_{q j}}+1\right\rangle \delta\left(\omega-\omega_{q j}\right) \\
\left\langle n_{\omega_{q j}}\right\rangle
\end{array}\right\},
\end{aligned}
$$

where $\mathbf{Q}$ and $\mathbf{G}$ are the total momentum transfer and the reciprocal lattice vector, respectively. $n_{\omega_{q j}}$ is the BoseEinstein distribution function. $f_{d}(\mathbf{Q})$ and $M_{d}$ are the atomic form factor and atomic mass of atom $d$, respectively. Wave function information enters $S(\mathbf{Q}, \omega)$ through the term $\mathbf{Q} \cdot \mathbf{e}_{d}^{j}(\mathbf{q})$.

For a topological chiral wave function, the three components of $\mathbf{e}_{d}^{j}(\mathbf{q})$ are complex numbers with different phase factors, which determine how atoms move out-of-phase with one another (see [34] for movies of the chiral atomic motions). The chirality results in a nontrivial interference between the displacements of different atoms, which is encoded in the large imaginary parts of $\mathbf{e}_{d}^{j}(\mathbf{q})$, i.e., chirality arises when the three components of $e_{d}^{j}(q)=\left[e_{d x}^{j}(q), e_{d y}^{j}(q), e_{d z}^{j}(q)\right]$ have phase difference. Although the phase of the sum $\sum_{d}(\ldots)$ is lost in $S(\mathbf{Q}, \omega)$ [see, Eq. (3)], the relative phase of wave-function components $\mathbf{e}_{d x}^{j}(\mathbf{q}), \mathbf{e}_{d y}^{j}(\mathbf{q}), \mathbf{e}_{d z}^{j}(\mathbf{q})$ that describe the wave-function chirality, are preserved. Thus, the chirality of the wave functions can strongly modify $S(\mathbf{Q}, \omega)$ [34]. To quantitatively show this effect, we simulate $S^{\mathrm{DFT}}(\mathbf{Q}, \omega), R(\mathbf{Q}, \omega)$, and $I(\mathbf{Q}, \omega)$ along the high-symmetry direction $R_{1}(-0.5,2.5,2.5)-\Gamma(0,3,3)-R_{2}(0.5,3.5,3.5)$ in Figs. 2(a) to 2(c), respectively. Here, $R(\mathbf{Q}, \omega)$ and $I(\mathbf{Q}, \omega)$ 

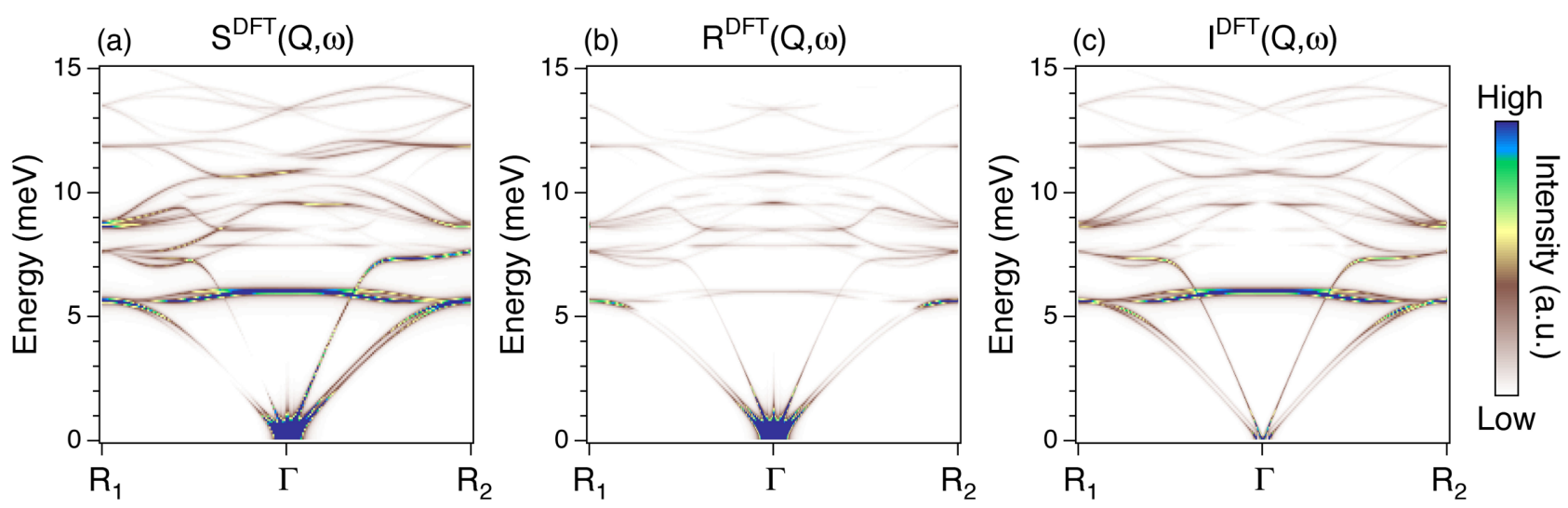

FIG. 2. DFT-calculated dynamical structural factor. (a)-(c) DFT-calculated $S^{\mathrm{DFT}}(\mathbf{Q}, \omega), R(\mathbf{Q}, \omega)$, and $I(\mathbf{Q}, \omega)$ along the high-symmetry $R_{1}(-0.5,2.5,2.5)-\Gamma(0,3,3)-R_{2}(0.5,3.5,3.5)$ direction, respectively. $R(\mathbf{Q}, \omega)$ and $I(\mathbf{Q}, \omega)$ considers only the real and imaginary parts of $\mathbf{e}_{d}^{j}(\mathbf{q})$. (a)-(c) are shown in the same colorscale.

are calculations of the dynamical structure factor shown in Eq. (3), but with $\mathbf{e}_{d}^{j}(\mathbf{q})$ replaced with its real part, $\operatorname{Re}\left[\mathbf{e}_{d}^{j}(\mathbf{q})\right]$, and imaginary part, $\operatorname{Im}\left[\mathbf{e}_{d}^{j}(\mathbf{q})\right]$, respectively. The chirality of the TQW near $6 \mathrm{meV}$ is evidenced by the large intensity contribution from the imaginary component of the wave function along the $R_{1}-\Gamma-R_{2}$ direction. As we will discuss in detail later in the text, this observation is a direct consequence of the TQW Hamiltonian [Eq. (1)]. Interestingly, we find that the threefold double Weyl modes at the $\Gamma$ point, including the acoustic phonons [7,12,34], display large intensity asymmetry in $S^{\mathrm{DFT}}(\mathbf{Q}, \omega)$ while they remain nearly symmetric in $R(\mathbf{Q}, \omega)$ and $I(\mathbf{Q}, \omega)$. This large asymmetry arises from the coexistence of real and imaginary components with comparable magnitude and hence have large interference effect. For the TQW mode along the [111] direction since $I(\mathbf{Q}, \omega) \gg$ $R(\mathbf{Q}, \omega)$, the interference term is small and keeps $S^{\mathrm{DFT}}(\mathbf{Q}, \omega)$ nearly symmetric.

To prove the existence of the topological chiral wave function in $\mathrm{BaPtGe}$, we present the experimental data of $S^{\exp }(\mathbf{Q}, \omega)$ that has been measured by IXS along the three high-symmetry directions, $X_{1}(0,3,2.5)-\Gamma-$ $X_{2}(0,3,3.5), M_{1}(0,2.5,2.5)-\Gamma-M_{2}(0,3.5,3.5), \quad$ and $\mathrm{R}_{1}(-0.5,2.5,2.5)-\Gamma-\mathrm{R}_{2}(0.5,3.5,3.5)$, in Figs. 3(a) to 3(c), respectively. To make a direct comparison with IXS, we show the experimental resolution-convoluted $S_{r}^{\mathrm{DFT}}(\mathbf{Q}, \omega)$ and $R_{r}(\mathbf{Q}, \omega)$ along the same momentum directions in Figs. 3(d) to 3(f) and Figs. 3(g) to 3(i). Here the subscript $r$ stands for resolution to distinguish from $S^{\mathrm{DFT}}(\mathbf{Q}, \omega)$ and $R(\mathbf{Q}, \omega) . S^{\exp }(\mathbf{Q}, \omega)$ and $S_{r}^{\mathrm{DFT}}(\mathbf{Q}, \omega)$ show excellent agreement, including the flatband near the TQW and the strong asymmetry of the threefold double Weyl optical and acoustic modes. This consistency is further confirmed by quantitative comparisons between experiment and theory shown in Fig. 4, where Figs. 4(a) to 4(c) displays the extracted $Q$-dependent peak positions from $S^{\exp }(\mathbf{Q}, \omega)$ and $S_{r}^{\mathrm{DFT}}(\mathbf{Q}, \omega)$ and Figs. 4(d) to 4(f) shows the extracted peak intensities near the TQW from $S^{\exp }(\mathbf{Q}, \omega), S_{r}^{\mathrm{DFT}}(\mathbf{Q}, \omega)$, and $R_{r}(\mathbf{Q}, \omega)$. Most interestingly, we find that wave functions near the TQW are primarily real along the $X_{1}-\Gamma-X_{2}$ and $M_{1}-\Gamma-M_{2}$ directions [Figs. 4(d) and 4(e)], in strong contrast to the $R_{1}-\Gamma-R_{2}$ direction [Fig. 4(f)], where the real-part of the wave function yields weak intensity near the TQW.
To understand the essential properties of the wave functions in different directions, we developed a simplified model that considers only Pt atoms in the unit cell, occupying the Wyckoff position $4 a$ [36] with locations $\mathbf{r}_{\mathrm{Pt} 1}=(c, c, c), \mathbf{r}_{\mathrm{Pt} 2}=\left(-c+\frac{1}{2},-c, c+\frac{1}{2}\right), \mathbf{r}_{\mathrm{Pt} 3}=(-c, c+$ $\left.\frac{1}{2},-c+\frac{1}{2}\right), \mathbf{r}_{\mathrm{Pt} 4}=\left(c+\frac{1}{2},-c+\frac{1}{2},-c\right)$. At the $\Gamma$ point, group theory dictates that we can describe the atomic motions in terms of three nondegenerate basis states with irreducible representations of $\Gamma_{1}^{(1)}, \Gamma_{2}^{(1)}, \Gamma_{3}^{(1)}$, and three threefold-degenerate basis states with irreducible representations of $\Gamma_{4}^{(3)}$ [36]. The basis states of $\Gamma_{2}^{(1)}$ and $\Gamma_{3}^{(1)}$ that forms the TQW can be derived by imposing chiral cubic crystal symmetry and time-reversal symmetry

$$
\begin{aligned}
\phi_{\Gamma_{2}} & =\left(e_{\mathrm{Pt} 1}^{\Gamma_{2}}, e_{\mathrm{Pt} 2}^{\Gamma_{2}}, e_{\mathrm{Pt} 3}^{\Gamma_{2}}, e_{\mathrm{Pt} 4}^{\Gamma_{2}}\right) \\
& =\frac{\left(1, \omega, \omega^{2},-1,-\omega, \omega^{2},-1, \omega,-\omega^{2}, 1,-\omega,-\omega^{2}\right)}{\sqrt{12}}, \\
\phi_{\Gamma_{3}} & =\left(e_{\mathrm{Pt} 1}^{\Gamma_{3}}, e_{\mathrm{Pt} 2}^{\Gamma_{3}}, e_{\mathrm{Pt} 3}^{\Gamma_{3}}, e_{\mathrm{Pt}}^{\Gamma_{3}}\right) \\
& =\frac{\left(1, \omega^{2}, \omega,-1,-\omega^{2}, \omega,-1, \omega^{2},-\omega, 1,-\omega^{2},-\omega\right)}{\sqrt{12}},
\end{aligned}
$$

where $\omega=e^{i 2 \pi / 3} . \phi_{\Gamma_{2}}$ and $\phi_{\Gamma_{3}}$ have opposite chirality following $\phi_{\Gamma_{2}}=\phi_{\Gamma_{3}}^{*}$. In the vicinity of the $\Gamma$ point, the $q$-dependent wave functions can be obtained by diagonalizing Eq. (1) in terms of our $\phi_{\Gamma_{2}}$ and $\phi_{\Gamma_{3}}$ basis states. Along the [100] and [011] directions, the diagonal components of Eq. (1) are zero, imposing purely real wave functions, $\psi_{1}=\left(e^{i \theta / 2} \phi_{\Gamma_{2}}+\right.$ $\left.e^{-i \theta / 2} \phi_{\Gamma_{3}}\right) / \sqrt{2}$ and $\psi_{2}=-i\left(e^{i \theta / 2} \phi_{\Gamma_{2}}-e^{-i \theta / 2} \phi_{\Gamma_{3}}\right) / \sqrt{2}$, where $\theta=\arg (B)$. In contrast, along the [111] direction, the off-diagonal components are zero yielding $\psi_{1}=\phi_{\Gamma_{2}}$ and $\psi_{2}=\phi_{\Gamma_{3}}$, which have large imaginary components. This model analysis thus demonstrates that the directional wave function observed in Figs. 3 and 4 is a direct consequence of the TQW Hamiltonian. This feature is unique among all experimentally investigated topological structures (see [34]). Figures $4(\mathrm{~g})$ and $4(\mathrm{~h})$ depict the chiral atomic motion of the TQW along the [111] direction. The results are calculated 

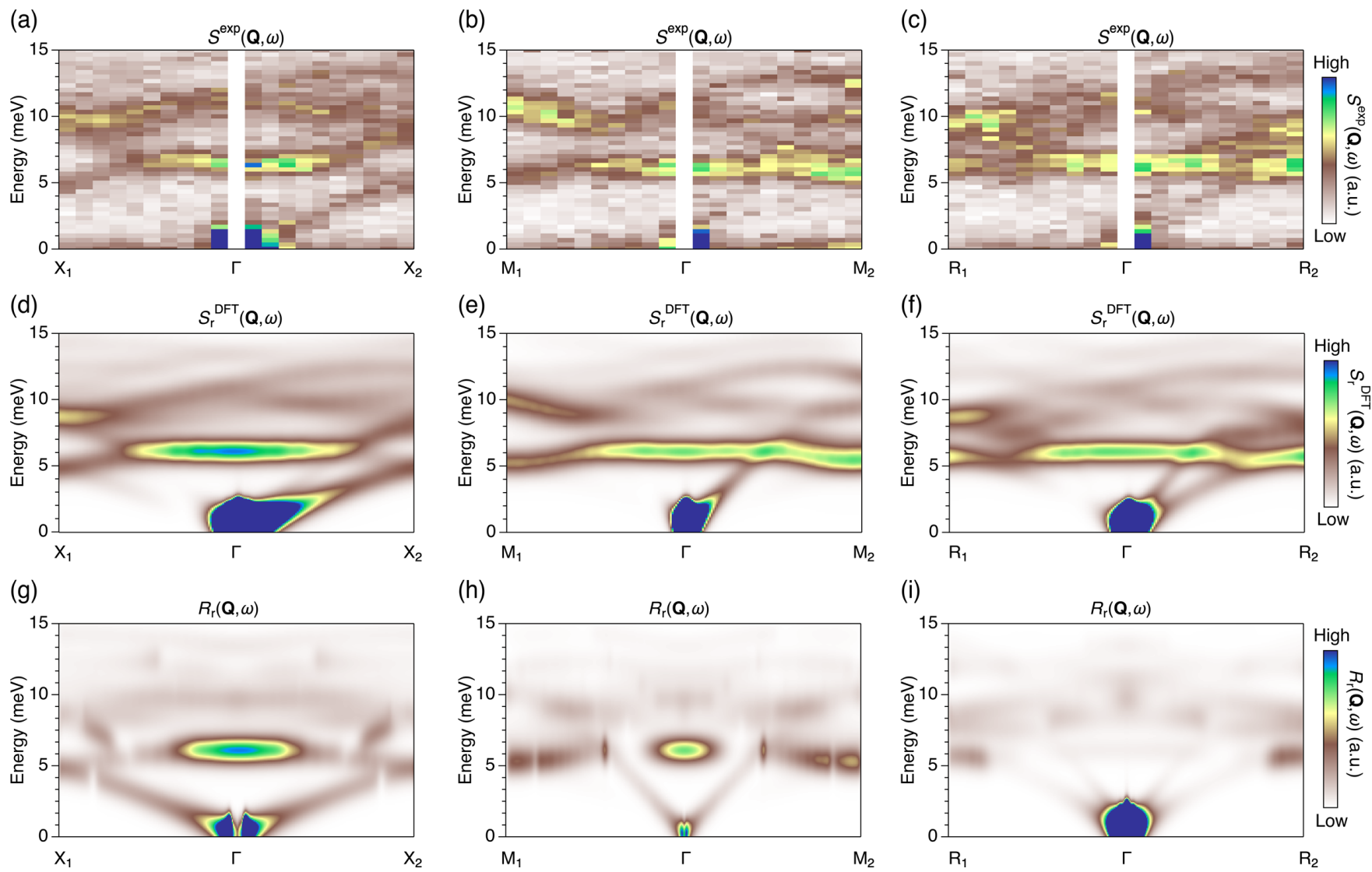

FIG. 3. Directional chiral wave function of the TQW. (a)-(c) Experimental $S^{\exp }(\mathbf{Q}, \omega)$ results along the three high-symmetry directions [001], [011], and [111], where $X_{1}=(0,3,2.5), X_{2}=(0,3,3.5), M_{1}=(0,2.5,2.5), M_{2}=(0,3.5,3.5), R_{1}=(-0.5,2.5,2.5), \quad R_{2}=$ $(0.5,3.5,3.5)$. Experimental resolution convoluted $S_{r}^{\mathrm{DFT}}(\mathbf{Q}, \omega), R_{r}(\mathbf{Q}, \omega)$ are shown in $(\mathrm{d})-(\mathrm{f})$ and $(\mathrm{g})-(\mathrm{i})$, respectively. The convolution function is determined by measuring the elastic scatting from plexiglass, which possesses a pseudo-voigt line shape with $\Delta E \sim 1.4$ meV. Panels (d)-(i) are shown in the same colorscale.

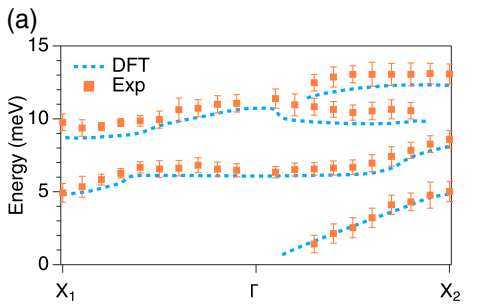

(d)

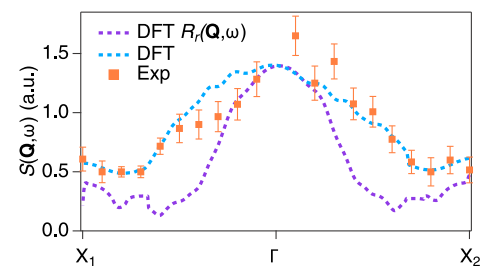

(b)

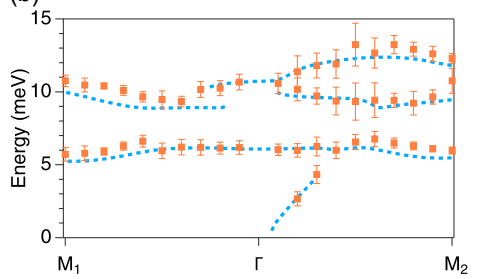

(e)

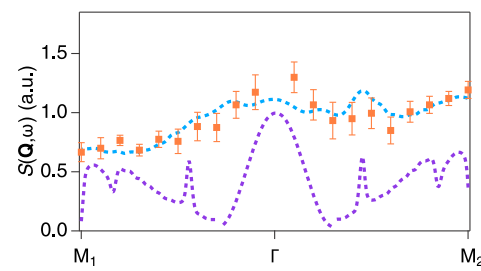

(c)

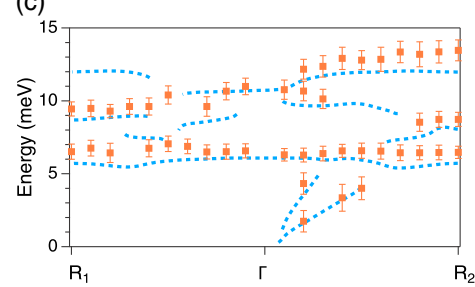

(f)

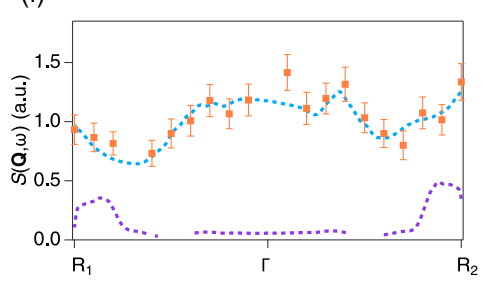

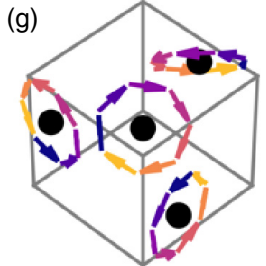

(g)

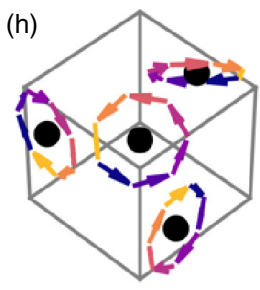

FIG. 4. $Q$-dependent phonon intensity. (a)-(c) The extracted peak intensities from the experimentally determined $S^{\exp }(\mathbf{Q}, \omega)$ (orange dots) and calculated $S_{r}^{\mathrm{DFT}}(\mathbf{Q}, \omega)$ (blue dashed lines) along [001], [011], and [111] directions, respectively. The curve near 6 meV corresponds to the TQW. (d)-(f) $Q$-dependent peak intensities of the TQW that are extracted from $S^{\exp }(\mathbf{Q}, \omega), S_{r}^{\mathrm{DFT}}(\mathbf{Q}, \omega), R_{r}^{\mathrm{DFT}}(\mathbf{Q}, \omega)$, respectively. Vertical bars in panels (a)-(c) denote errors of the peak positions estimated based on the energy resolution and counting statistics. Error bars in panels (d)-(f) denote one standard deviation assuming Poissonian counting statistics in the measured $S^{\exp }(\mathbf{Q}, \omega)$ intensity. Panels (g) and (h) depict the chiral atomic motion of the TQW with $C=+4$ and -4 , respectively. The results are calculated from the simplified model along the [111] direction. Colored arrows are pointing to the directions of Pt motions at a given time. Chirality is determined by the (g) clockwise and (h) anticlockwise motions. 
from the simplified model with the colored arrows representing the directions of Pt motions at a given time. The opposite chirality yields opposite $\mathrm{Pt}$ motions, in agreement with our numerical calculation of BaPtGe (see Supplemental Materials [34] for movies of the chiral atomic motions). Finally, we note that the intensity asymmetry of the threefold double Weyl modes is also due to chiral wave functions as directly evidenced from our numerical calculations (Fig. 2). However, this finite $\mathbf{q}$ effect is beyond our simplified model where the effective Hamiltonian of threefold double Weyl contains only linear terms (see [34]). Slightly away from the $\Gamma$ point, higher-order terms become important and the linear term approximation fails to describe the phonon excitations. For the TQW, the linear term is absent, and Eq. (1) with its square and cubic terms remains valid for an extend momentum space.

Similar to the electronic topological Hall effect [37], the intrinsic thermal Hall effect is also proportional to Chern number $C[38,39]$. The observed TQW may thus provide an ideal platform to explore intrinsic thermal Hall effect [40,41]. More importantly, our result paves a new route to uncover topological excitations through bulk wave functions. Similar attempts have been made in the study of topological electronic structures via angle-resolved photoemission spectroscopy (ARPES) [42-44]. While the momentum-dependent ARPES intensity provides important clue on the symmetry of the surface wave function, the presence of surface Coulomb potentials makes it difficult to calculate the ARPES intensity from first principles [42]. Our approach, based on quantitative intensity and dispersion comparisons between $S^{\exp }(\mathbf{Q}, \omega)$, $S^{\mathrm{DFT}}(\mathbf{Q}, \omega), R(\mathbf{Q}, \omega)$, and $I(\mathbf{Q}, \omega)$, can also be extended to inelastic neutron scattering to explore the bulk wave functions of topological phonons and magnons, which may play a crucial role in the giant thermal Hall effect observed in several correlated quantum materials $[39,45]$.

The recent discoveries of giant thermal Hall effect in the cuprate high- $T_{c}$ superconductors $[45,46]$ and Kitaev quantum spin liquid candidate $\alpha-\mathrm{RuCl}_{3}[47,48]$ generate great interest on the novel topological nature of these quantum materials, including fractional excitations [49,50] and topological bosonic excitations [51-53]. Indeed, chiral phonons have been argued as the driving force of the thermal Hall effect in the cuprates $[45,46]$. Our result provides an experimental protocol to uncover the nature of these novel thermal transport properties and shed light on topological excitations in correlated quantum materials.

This research at Oak Ridge National Laboratory (ORNL) was sponsored by the U.S. Department of Energy, Office of Science, Basic Energy Sciences, Materials Sciences and Engineering Division (IXS data analysis) and by the Laboratory Directed Research and Development Program of ORNL, managed by UT-Battelle, LLC, for the U.S. Department of Energy (IXS experiment). Part of IXS data interpretation work at Brookhaven National Laboratory was supported by the U.S. DOE, Office of Science, Office of Basic Energy Sciences, Materials Sciences, and Engineering Division under Contract No. DE- SC0012704 and by the U.S. DOE, Office of Basic Energy Sciences, Early Career Award Program under Award No. 1047478. This research used resources of the Advanced Photon Source, a U.S. Department of Energy (DOE) Office of Science User Facility, operated for the DOE Office of Science by Argonne National Laboratory under Contract No. DE-AC02-06CH11357. Extraordinary facility operations were supported, in part, by the DOE Office of Science through the National Virtual Biotechnology Laboratory, a consortium of DOE national laboratories focused on the response to COVID-19, with funding provided by the Coronavirus CARES Act. T.T.Z. and S.M. acknowledge the supports from Tokodai Institute for Element Strategy (TIES) funded by MEXT Elements Strategy Initiative to Form Core Research Center. T.T.Z. also acknowledge the support by Japan Society for the Promotion of Science (JSPS), KAKENHI Grant No. 21K13865. S.M. also acknowledges support by JSPS KAKENHI Grant No. JP18H03678. H.C.L. acknowledges the supports from the National Key R\&D Program of China (Grants No. 2016YFA0300504 and No. 2018YFE0202600), the National Natural Science Foundation of China (Grants No. 11774423 and No. 11822412), the Fundamental Research Funds for the Central Universities, and the Research Funds of Renmin University of China (Grants No. 18XNLG14 and No. 19XNLG17).
[1] X. Wan, A. M. Turner, A. Vishwanath, and S. Y. Savrasov, Topological semimetal and fermi-arc surface states in the electronic structure of pyrochlore iridates, Phys. Rev. B 83, 205101 (2011).

[2] H. Weng, C. Fang, Z. Fang, B. A. Bernevig, and X. Dai, Weyl Semimetal Phase in Noncentrosymmetric TransitionMetal Monophosphides, Phys. Rev. X 5, 011029 (2015).

[3] J. Xiong, S. K. Kushwaha, T. Liang, J. W. Krizan, M. Hirschberger, W. Wang, R. J. Cava, and N. P. Ong, Evidence for the chiral anomaly in the dirac semimetal $\mathrm{Na}_{3} \mathrm{Bi}$, Science 350, 413 (2015).

[4] G. Chang, S.-Y. Xu, B. J. Wieder, D. S. Sanchez, S.-M. Huang, I. Belopolski, T.-R. Chang, S. Zhang, A. Bansil, H. Lin, and M. Z. Hasan, Unconventional Chiral Fermions and Large Topological Fermi Arcs in Rhsi, Phys. Rev. Lett. 119, 206401 (2017).
[5] A. A. Soluyanov, D. Gresch, Z. Wang, Q. Wu, M. Troyer, X. Dai, and B. A. Bernevig, Type-ii Weyl semimetals, Nature (London) 527, 495 (2015).

[6] B. Bradlyn, J. Cano, Z. Wang, M. G. Vergniory, C. Felser, R. J. Cava, and B. A. Bernevig, Beyond dirac and Weyl fermions: Unconventional quasiparticles in conventional crystals, Science 353, aaf5037 (2016).

[7] T. Zhang, Z. Song, A. Alexandradinata, H. Weng, C. Fang, L. Lu, and Z. Fang, Double-Weyl Phonons in Transition-Metal Monosilicides, Phys. Rev. Lett. 120, 016401 (2018).

[8] L. Lu, L. Fu, J. D. Joannopoulos, and M. Soljačić, Weyl points and line nodes in gyroid photonic crystals, Nat. Photonics 7, 294 (2013).

[9] Q. Lin, M. Xiao, L. Yuan, and S. Fan, Photonic Weyl point in a two-dimensional resonator lattice with a synthetic frequency dimension, Nat. Commun. 7, 13731 (2016). 
[10] M. Xiao, Q. Lin, and S. Fan, Hyperbolic Weyl Point in Reciprocal Chiral Metamaterials, Phys. Rev. Lett. 117, 057401 (2016).

[11] F.-Y. Li, Y.-D. Li, Y. B. Kim, L. Balents, Y. Yu, and G. Chen, Weyl magnons in breathing pyrochlore antiferromagnets, Nat. Commun. 7, 12691 (2016).

[12] H. Miao, T. T. Zhang, L. Wang, D. Meyers, A. H. Said, Y. L. Wang, Y. G. Shi, H. M. Weng, Z. Fang, and M. P. M. Dean, Observation of Double Weyl Phonons in Parity-Breaking Fesi, Phys. Rev. Lett. 121, 035302 (2018).

[13] T. T. Zhang, H. Miao, Q. Wang, J. Q. Lin, Y. Cao, G. Fabbris, A. H. Said, X. Liu, H. C. Lei, Z. Fang, H. M. Weng, and M. P. M. Dean, Phononic Helical Nodal Lines with $\mathcal{P} \mathcal{T}$ Protection in mob $_{2}$, Phys. Rev. Lett. 123, 245302 (2019).

[14] B. W. Xia, R. Wang, Z. J. Chen, Y. J. Zhao, and H. Xu, Symmetry-Protected Ideal Type-ii Weyl Phonons in CdTe, Phys. Rev. Lett. 123, 065501 (2019).

[15] Q.-B. Liu, Y. Qian, H.-H. Fu, and Z. Wang, Symmetry-enforced Weyl phonons, npj Comput. Mater. 6, 95 (2020).

[16] B. Q. Lv, H. M. Weng, B. B. Fu, X. P. Wang, H. Miao, J. Ma, P. Richard, X. C. Huang, L. X. Zhao, G. F. Chen, Z. Fang, X. Dai, T. Qian, and H. Ding, Experimental Discovery of Weyl Semimetal Taas, Phys. Rev. X 5, 031013 (2015).

[17] S.-Y. Xu, I. Belopolski, N. Alidoust, M. Neupane, G. Bian, C. Zhang, R. Sankar, G. Chang, Z. Yuan, C.-C. Lee, S.-M. Huang, H. Zheng, J. Ma, D. S. Sanchez, B. K. Wang, A. Bansil, F. Chou, P. P. Shibayev, H. Lin, S. Jia, and M. Zahid Hasan, Discovery of a Weyl fermion semimetal and topological fermi arcs, Science 349, 613 (2015).

[18] L. X. Yang, Z. K. Liu, Y. Sun, H. Peng, H. F. Yang, T. Zhang, B. Zhou, Y. Zhang, Y. F. Guo, M. Rahn, D. Prabhakaran, Z. Hussain, S.-K. Mo, C. Felser, B. Yan, and Y. L. Chen, Weyl semimetal phase in the non-centrosymmetric compound taas, Nat. Phys. 11, 728 (2015).

[19] L. Lu, Z. Wang, D. Ye, L. Ran, L. Fu, J. D. Joannopoulos, and M. Soljačić, Experimental observation of Weyl points, Science 349, 622 (2015).

[20] K. Deng, G. Wan, P. Deng, K. Zhang, S. Ding, E. Wang, M. Yan, H. Huang, H. Zhang, Z. Xu, J. Denlinger, A. Fedorov, H. Yang, W. Duan, H. Yao, Y. Wu, S. Fan, H. Zhang, X. Chen, and S. Zhou, Experimental observation of topological fermi arcs in type-ii Weyl semimetal MoTe 2 , Nat. Phys. 12, 1105 (2016).

[21] A. Tamai, Q. S. Wu, I. Cucchi, F. Y. Bruno, S. Riccò, T. K. Kim, M. Hoesch, C. Barreteau, E. Giannini, C. Besnard, A. A. Soluyanov, and F. Baumberger, Fermi Arcs and Their Topological Character in the Candidate Type-ii Weyl Semimetal $\mathrm{MoTe}_{2}$, Phys. Rev. X 6, 031021 (2016).

[22] W.-J. Chen, M. Xiao, and C. T. Chan, Photonic crystals possessing multiple Weyl points and the experimental observation of robust surface states, Nat. Commun. 7, 13038 (2016).

[23] B. Yang, Q. Guo, B. Tremain, R. Liu, L. E. Barr, Q. Yan, W. Gao, H. Liu, Y. Xiang, J. Chen, C. Fang, A. Hibbins, L. Lu, and S. Zhang, Ideal Weyl points and helicoid surface states in artificial photonic crystal structures, Science 359, 1013 (2018).

[24] Q.-Q. Yuan, L. Zhou, Z.-C. Rao, S. Tian, W.-M. Zhao, C.-L. Xue, Y. Liu, T. Zhang, C.-Y. Tang, Z.-Q. Shi, Z.-Y. Jia, H. Weng, H. Ding, Y.-J. Sun, H. Lei, and S.-C. Li, Quasiparticle interference evidence of the topological fermi arc states in chiral fermionic semimetal cosi, Sci. Adv. 5, eaaw9485 (2019).

[25] H. Jia, R. Zhang, W. Gao, Q. Guo, B. Yang, J. Hu, Y. Bi, Y. Xiang, C. Liu, and S. Zhang, Observation of chiral zero mode in inhomogeneous three-dimensional Weyl metamaterials, Science 363, 148 (2019).

[26] P. Hosur, S. A. Parameswaran, and A. Vishwanath, Charge Transport in Weyl Semimetals, Phys. Rev. Lett. 108, 046602 (2012).

[27] H.-J. Kim, K.-S. Kim, J.-F. Wang, M. Sasaki, N. Satoh, A. Ohnishi, M. Kitaura, M. Yang, and L. Li, Dirac Versus Weyl Fermions in Topological Insulators: Adler-Bell-Jackiw Anomaly in Transport Phenomena, Phys. Rev. Lett. 111, 246603 (2013).

[28] S. A. Parameswaran, T. Grover, D. A. Abanin, D. A. Pesin, and A. Vishwanath, Probing the Chiral Anomaly with Nonlocal Transport in Three-Dimensional Topological Semimetals, Phys. Rev. X 4, 031035 (2014).

[29] Z. Song and X. Dai, Hear the Sound of Weyl Fermions, Phys. Rev. X 9, 021053 (2019).

[30] J. Xiang, S. Hu, Z. Song, M. Lv, J. Zhang, L. Zhao, W. Li, Z. Chen, S. Zhang, J.-T. Wang, Y.-f. Yang, X. Dai, F. Steglich, G. Chen, and P. Sun, Giant Magnetic Quantum Oscillations in the Thermal Conductivity of Taas: Indications of Chiral Zero Sound, Phys. Rev. X 9, 031036 (2019).

[31] W. Yao, C. Li, L. Wang, S. Xue, Y. Dan, K. Iida, K. Kamazawa, K. Li, C. Fang, and Y. Li, Topological spin excitations in a threedimensional antiferromagnet, Nat. Phys. 14, 1011 (2018).

[32] T. Zhang, R. Takahashi, C. Fang, and S. Murakami, Twofold quadruple Weyl nodes in chiral cubic crystals, Phys. Rev. B 102, 125148 (2020).

[33] J.-X. Yin, N. Shumiya, S. Mardanya, Q. Wang, S. S. Zhang, H.-J. Tien, D. Multer, Y. Jiang, G. Cheng, N. Yao, S. Wu, D. Wu, L. Deng, Z. Ye, R. He, G. Chang, Z. Liu, K. Jiang, Z. Wang, T. Neupert, A. Agarwal, T.-R. Chang, C.-W. Chu, H. Lei, and M. Zahid Hasan, Fermion-boson many-body interplay in a frustrated kagome paramagnet, Nat. Commun. 11, 4003 (2020).

[34] See Supplemental Material at http://link.aps.org/supplemental/ 10.1103/PhysRevB.103.184301 for more details on the pseudospin properties.

[35] Z. Rao, H. Li, T. Zhang, S. Tian, C. Li, B. Fu, C. Tang, L. Wang, Z. Li, W. Fan, J. Li, Y. Huang, Z. Liu, Y. Long, C. Fang, H. Weng, Y. Shi, H. Lei, Y. Sun, T. Qian, and H. Ding, Observation of unconventional chiral fermions with long fermi arcs in $\mathrm{CoSi}$, Nature (London) 567, 496 (2019).

[36] M. I. Aroyo, J. M. Perez-Mato, C. Capillas, E. Kroumova, S. Ivantchev, G. Madariaga, A. Kirov, and H. Wondratschek, Bilbao crystallographic server: I. databases and crystallographic computing programs, Z. Kristallogr. 221, 15 (2006).

[37] N. Nagaosa, J. Sinova, S. Onoda, A. H. MacDonald, and N. P. Ong, Anomalous Hall effect, Rev. Mod. Phys. 82, 1539 (2010).

[38] T. Qin, J. Zhou, and J. Shi, Berry curvature and the phonon Hall effect, Phys. Rev. B 86, 104305 (2012).

[39] J.-Y. Chen, S. A. Kivelson, and X.-Q. Sun, Enhanced Thermal Hall Effect in Nearly Ferroelectric Insulators, Phys. Rev. Lett. 124, 167601 (2020).

[40] V. Cvetkovic and O. Vafek, Berry phases and the intrinsic thermal Hall effect in high-temperature cuprate superconductors, Nat. Commun. 6, 6518 (2015).

[41] S. A. Owerre, Topological thermal Hall effect in frustrated kagome antiferromagnets, Phys. Rev. B 95, 014422 (2017).

[42] X.-P. Wang, P. Richard, Y.-B. Huang, H. Miao, L. Cevey, N. Xu, Y.-J. Sun, T. Qian, Y.-M. Xu, M. Shi, J.-P. Hu, X. Dai, and H. Ding, Orbital characters determined from fermi surface 
intensity patterns using angle-resolved photoemission spectroscopy, Phys. Rev. B 85, 214518 (2012).

[43] Y. Cao, J. A. Waugh, X.-W. Zhang, J.-W. Luo, Q. Wang, T. J. Reber, S. K. Mo, Z. Xu, A. Yang, J. Schneeloch, G. D. Gu, M. Brahlek, N. Bansal, S. Oh, A. Zunger, and D. S. Dessau, Mapping the orbital wavefunction of the surface states in threedimensional topological insulators, Nat. Phys. 9, 499 (2013).

[44] Y. Liu, G. Bian, T. Miller, and T.-C. Chiang, Visualizing Electronic Chirality and Berry Phases in Graphene Systems Using Photoemission with Circularly Polarized Light, Phys. Rev. Lett. 107, 166803 (2011).

[45] G. Grissonnanche, S. Thériault, A. Gourgout, M.-E. Boulanger, E. Lefrançois, A. Ataei, F. Laliberté, M. Dion, J.-S. Zhou, S. Pyon, T. Takayama, H. Takagi, N. Doiron-Leyraud, and L. Taillefer, Chiral phonons in the pseudogap phase of cuprates, Nat. Phys. 16, 1108 (2020).

[46] G. Grissonnanche, A. Legros, S. Badoux, E. Lefrançois, V. Zatko, M. Lizaire, F. Laliberté, A. Gourgout, J.-S. Zhou, S. Pyon, T. Takayama, H. Takagi, S. Ono, N. Doiron-Leyraud, and L. Taillefer, Giant thermal Hall conductivity in the pseudogap phase of cuprate superconductors, Nature (London) 571, 376 (2019).

[47] Y. Kasahara, T. Ohnishi, Y. Mizukami, O. Tanaka, S. Ma, K. Sugii, N. Kurita, H. Tanaka, J. Nasu, Y. Motome, T. Shibauchi, and Y. Matsuda, Majorana quantization and half-integer thermal quantum Hall effect in a kitaev spin liquid, Nature (London) 559, 227 (2018).

[48] Y. Kasahara, K. Sugii, T. Ohnishi, M. Shimozawa, M. Yamashita, N. Kurita, H. Tanaka, J. Nasu, Y. Motome, T. Shibauchi, and Y. Matsuda, Unusual Thermal Hall Effect in a Kitaev Spin Liquid Candidate $\alpha-\operatorname{rucl}_{3}$, Phys. Rev. Lett. 120, 217205 (2018).

[49] M. Ye, G. B. Halász, L. Savary, and L. Balents, Quantization of the Thermal Hall Conductivity at Small Hall Angles, Phys. Rev. Lett. 121, 147201 (2018).

[50] Y. Vinkler-Aviv and A. Rosch, Approximately Quantized Thermal Hall Effect of Chiral Liquids Coupled to Phonons, Phys. Rev. X 8, 031032 (2018).

[51] P. A. McClarty, X.-Y. Dong, M. Gohlke, J. G. Rau, F. Pollmann, R. Moessner, and K. Penc, Topological magnons in kitaev magnets at high fields, Phys. Rev. B 98, 060404(R) (2018).

[52] J. H. Han, J.-H. Park, and P. A. Lee, Consideration of thermal Hall effect in undoped cuprates, Phys. Rev. B 99, 205157 (2019).

[53] R. Samajdar, M. S. Scheurer, S. Chatterjee, H. Guo, C. Xu, and S. Sachdev, Enhanced thermal hall effect in the square-lattice néel state, Nat. Phys. 15, 1290 (2019). 